\section{$\underset{\text { \& migrations }}{\text { hommes }}$}

\section{Hommes \& migrations}

Revue française de référence sur les dynamiques

migratoires

$1316 \mid 2017$

L'islam en Europe

\title{
2006-2016, 10 ans d'aumônerie militaire du culte musulman
}

Bilan et perspectives

\section{Elyamine Settoul}

\section{OpenEdition}

Journals

Édition électronique

URL : http://journals.openedition.org/hommesmigrations/3804

DOI : 10.4000/hommesmigrations.3804

ISSN : 2262-3353

Éditeur

Musée national de l'histoire de l'immigration

Édition imprimée

Date de publication : 1 mars 2017

Pagination : 109-117

ISBN : 978-2-919040-37-7

ISSN : 1142-852X

Référence électronique

Elyamine Settoul, « 2006-2016, 10 ans d'aumônerie militaire du culte musulman », Hommes \& migrations [En ligne], 1316 | 2017, mis en ligne le 01 mars 2020, consulté le 17 septembre 2020. URL : http://journals.openedition.org/hommesmigrations/3804 ; DOI : https://doi.org/10.4000/ hommesmigrations.3804 


\title{
2006-2016, 10 ANS D'AUMÔNERIE MILITAIRE DU CULTE MUSULMAN BILAN ET PERSPECTIVES
}

Par ELYAMINE SETTOUL, chercheur associé en science politique à l'Institut de recherche stratégique de l'École militaire.

\begin{abstract}
L'aumônerie militaire du culte musulman est une création récente en France. Structurée depuis une décennie au sein de l'armée française, la présence d'aumôniers militaires musulmans constitue un symbole fort, de la part de la grande muette, dans la reconnaissance de la diversité des croyances de ses troupes. Ces aumôniers traduisent également la volonté d'encadrer la pratique de l'islam dans les régiments. Si la religion musulmane demeure un sujet sensible dans la société française, objet de discriminations et de préjugés, les valeurs de soumission et de respect qu'elle prône participent à renforcer l'esprit de corps dans l'armée, fondé sur l'unité d'une République laïque.
\end{abstract}

L'instauration d'une aumônerie militaire musulmane en 2006 par la ministre de la Défense Michèle Alliot-Marie constitue une initiative historique qui, de fait, met fin à une situation anticonstitutionnelle pour les soldats français partageant cette confession. En effet, ce traitement différencié s'inscrit de fait en discordance avec le premier article de la Constitution de 1958 qui institue le fait que " la République assure l'égalité devant la loi de tous les citoyens sans distinction d'origine, de race ou de religion. Elle respecte toutes les croyances ». Cette normalisation apparaît comme un phénomène relativement récent lorsqu'on prend en considération le fait que l'institution compte une présence massive de musulmans depuis la période de conquête coloniale de l'Afrique du Nord au XIX siècle. Audelà de cette émergence tardive, l'analyse de la mise en place d'une aumônerie militaire musulmane dans le contexte français constitue un domaine de recherche intéressant à plusieurs titres. Outre qu'elle dispose de la plus importante communauté musulmane d'Europe, la société française entretient des relations historiques passionnelles et tumultueuses avec la notion de laïcité1. Les nombreuses controverses et querelles juridiques qui nourrissent les débats publics depuis les années 1990 en sont 
l'une des illustrations. Enfin, la tradition militaire française a toujours mis l'accent sur la notion de représentativité sociale.

À travers la mise en place et l'extension progressive du système de conscription depuis la fin du XIX siècle, les armées ont développé une relation quasi-fusionnelle avec la société civile. Cette dernière symbolisant encore dans la mythologie républicaine post-révolutionnaire ${ }^{2}$ la " nation en armes ». Bien que totalement professionnalisée depuis 1996, ce référentiel idéologique fondateur demeure encore très présent dans l'imaginaire et le corpus des valeurs militaires françaises. Preuve de la prégnance de cette matrice, un rapport sénatorial de 1996 consacrée à la professionnalisation des armées signalait explicitement l'importance de recruter parmi les communautés issues de l'immigration afin de pallier aux besoins en ressources humaines tout en assurant un haut niveau de représentativité sociologique ${ }^{3}$. La conjonction de ces multiples spécificités d'ordres sociologique, juridique et idéologique rend la configuration française particulièrement originale. Partant de ces différents constats, la présente contribution vise à explorer les modalités par lesquelles s'est élaborée l'institutionnalisation du fait musulman au sein de la sphère militaire. La compréhension de cette reconnaissance institutionnelle implique de retracer au préalable les conditions historiques de la présence musulmane sous les drapeaux français.

\section{Présence ancienne et organisation conjoncturelle du culte musulman}

Massivement enrôlés avec la conquête de l'Afrique du Nord en 1830, les autochtones musulmans ont constitué des régiments de tirailleurs et de spahis particulièrement efficaces pour l'expansion coloniale. Habitués au climat local et connaisseurs des nouveaux espaces à conquérir, ils ont représenté pour les armées françaises une ressource humaine considérable tout au long des XIX et $\mathrm{XX}^{\mathrm{e}}$ siècles. Ainsi, lors de la Première Guerre mondiale, les contingents coloniaux (combattants et travailleurs) s'élevèrent à 818000 individus ${ }^{4}$. Pour cette raison, la question de l'islam a fait l'objet d'une attention précoce. L'organisation du culte musulman a été rythmée par diverses tentatives, des concessions et des mesures plus ou moins officieuses. Dans un article consacré à ce sujet, Xavier Boniface retrace la lente gestation de l'aumônerie musulmane, du début de la Grande Guerre à 1962. Il rapporte que les préoccupations religieuses des tirailleurs (salles de prières, repas sans porc...) étaient déjà prises en compte par les autorités militaires surtout lors des périodes de conflit où « la foi représente bien souvent l'ultime bastion défensif " ". Un premier aumônier musulman fut nommé en 1920 et affecté auprès des unités nord-africaines détachées en Syrie, mais les élites militaires de l'époque jugèrent son action défavorable aux intérêts français et le licencièrent trois ans plus tard. Bien que l'idée de créer une aumônerie musulmane ait par la suite persisté dans l'esprit de certains décideurs militaires, elle ne dépassa jamais le stade de projet. Les adversaires de cette initiative justifiaient leur refus en prétextant que le concept d'aumônerie était étranger à la tradition islamique et qu'un tel projet risquait, de plus, de favoriser le prosélytisme religieux au sein des «troupes noires aux croyances fétichistes ». Or " la conversion des fétichistes à l'islam ne présente que des dangers pour la puissance française $e^{6}$ ». À défaut d'une aumônerie musulmane, l'état-major instaura par le décret 
du 14 mai 1940 un service d'assistance religieuse à l'intention des musulmans en service dans la métropole. Celui-ci visait à entretenir et fortifier le moral des troupes qui comprenaient difficilement les inégalités de traitement qu'elles subissaient, notamment en termes de soldes et d'avancement de carrières. Avec l'ordonnance du Général De Gaulle de 1943, quinze « imams civils » furent recrutés par l'armée de la France libre, afin de renforcer l'esprit patriotique des tirailleurs et réaffirmer le caractère juste de la guerre qu'elle livrait (Just War Theory). Ces aumôniers à part entière n'eurent le droit d'exercer leur fonction que pendant la seule période de la guerre.

Au lendemain de la Seconde Guerre mondiale, l'idée d'une organisation officielle du culte est peu à peu abandonnée et la prise en compte des préoccupations religieuses devint la mission d'officiers ou de sous-officiers désignés par le commandement. La crainte d'un noyautage des unités par des imams qui inciteraient à une plus grande observance des rites religieux demeurait malgré tout encore prégnante. Les conflits liés à la décolonisation accentuèrent encore un peu plus le caractère " sensible » de cette question puisque, désormais, il s'agissait de composer avec un ennemi professant la même religion. Dès lors, le commandement refusa toute intrusion d'imams, a priori suspects de subversion. Après la fin de la Guerre d'Algérie, qui sonne le glas de l'Empire colonial français, la mission de l'étatmajor consiste à répondre aux préoccupations des conscrits. Il s'agit là d'un tournant sociopolitique majeur car, désormais, le fait islamique ne concerne plus des sujets de l'Empire enrôlés dans des formations militaires quasi-monoconfessionnelles, mais des citoyens français effectuant leur service national ou des volontaires engagés dans des unités où ils représentent une minorité (au sens numérique). La prise en compte des prescriptions religieuses fut officieusement déléguée à des militaires dont la mission consistait principalement à veiller au respect des rites funéraires pour les soldats morts au combat $^{7}$. L'ancrage d'une immigration de travail en provenance de pays majoritairement musulmans va inéluctablement réactualiser cette question de la gestion de la pluralité religieuse au sein des armées et contraindre le politique à se positionner.

\section{Le service national et les difficultés d'intégration des secondes générations issues de l'immigration}

L'un des principaux facteurs ayant favorisé la réflexion et l'organisation du culte musulman au sein des armées est de nature fonctionnelle. La sédentarisation des grandes vagues d'immigration industrielle des années 1960 va naturellement laisser place à des secondes générations. Issues pour la plupart de l'immigration maghrébine et, dans une moindre mesure, africaine, ces nouvelles générations vont venir rejoindre les contingents annuels de conscrits. Cette transformation sociologique souleva un certain nombre de questionnements auprès du haut-commandement qui lança, dès 1990, l'une des toutes premières études françaises consacrée à la question de la diversité culturelle au sein d'une grande institution publique.

La prise en compte des prescriptions religieuses fut officieusement déléguée à des militaires dont la mission consistait principalement à veiller au respect des rites funéraires pour les soldats morts au combat. Rédigée sous la direction du Colonel Yves Biville, l'étude "Armées et population à problèmes d'intégration : le cas des jeunes Français d'origine maghrébine » dresse un état des lieux de l'expérience de ces conscrits. Menée par deux militaires et un civil, l'enquête se voulait un outil de compréhension pour les cadres désireux d'identifier les difficultés d'insertion des jeunes Français d'origine maghrébine (JFOM). S’appuyant sur un échantillon composé de 550 personnes, les auteurs du rapport mettaient en exergue le fait que les JFOM conjuguaient plusieurs types de difficultés. Du point de vue religieux, l'absence d'aumônerie 
islamique et la faible prise en compte des prescriptions liées à l'exercice de leur foi (ramadan, menu sans porc...) étaient vécues comme une forme de stigmatisation symbolique. De plus, ce groupe se caractérisait par un niveau scolaire globalement plus faible que la moyenne des conscrits, et $95 \%$ d'entre eux occupaient des postes subalternes de soldats de deuxième classe. L'institution militaire tendait davantage à reproduire les inégalités du milieu civil quà aider socialement ces groupes sociaux défavorisés et relégués. Les enquêteurs insistaient fortement sur le malaise identitaire de ces conscrits qui, malgré de fortes attentes initiales, avaient le sentiment de ne pas " trouver leur place " au sein des armées. Cumulés à la non-reconnaissance institutionnelle de leur religion, ces handicaps sociaux favorisaient, selon les auteurs, un repli communautaire vers l'identité musulmane. Cette problématique était accentuée par le fait que les militaires de confession catholique jouissaient, à l'inverse, d'un certain nombre de facilités en termes de pratiques et de célébrations religieuses. La plupart des régiments français fêtent des saints patrons tirés du sanctoral catholique et développent une importante vie sociale et culturelle autour du fait reli-
La facilitation de la pratique religieuse était très variable d'un régiment à l'autre et, de manière globale, peu d'évolutions avaient été constatées depuis la période de la conscription gieux (organisation de pèlerinages, messes dominicales...). Un haut gradé des armées françaises témoigne ainsi de cette réalité : "Cette influence est perceptible à travers quelques exemples vécus. Dans les années 1990 déjà, un ancien chef de corps affichait sa fierté d'avoir amené au baptême trois de ses soldats lors d'une de ses OPEX (opérations extérieures). Dans un séminaire de cadres au collège interarmées de défense (CID), j'ai eu une certaine surprise à entendre deux officiers supérieurs d'armées différentes se présenter comme officiers catholiques devant leur hiérarchie et leurs camarades sans susciter une quelconque réaction. À Saint-Cyr Coëtquidan, l'expression d'une foi ardente en uniforme a été régulière- ment signalée parmi les élèves officiers et a inquiété le commandement. C'est aussi cet autre officier qui fait remettre en état une chapelle à Pristina au Kosovo. J'ai, enfin, ce cas d'un officier de confession israélite se sentant écarté par ses pairs, et ce dernier exemple rapporté de cette épouse d'officier protestant se sentant mise à l'écart par une partie des épouses catholiques [...]. Ces exemples montrent également une grande tolérance interne sur l'expression de la foi catholique au sein de notre institution, en contradiction avec sa laïcité institutionnelle. Je me demande d'ailleurs si le seul lieu où cela est possible dans l'administration publique, ne reste justement pas l'institution militaire. ${ }^{8}$ "

\section{Entre persistance des discriminations religieuses et crainte du repli confessionnel}

Ce " deux poids deux mesures » tendait à générer des crispations et à alimenter un sentiment de frustration auprès des militaires de confession musulmane, notamment à l'occasion de missions qui pouvaient parfois durer plusieurs mois (opérations extérieures, missions en mer). Il favorisait également le regroupement de soldats de culture musulmane, ce qui pouvait être une source de questionnements voire d'inquiétude pour les membres du commandement ${ }^{9}$. La mise en œuvre de la circulaire Joxe en mai 1992 répondait au souci de mieux prendre en considération les besoins du personnel militaire musulman en leur permettant par exemple de prendre des jours de congés lors des fêtes religieuses (aïd), de bénéficier de menus halal ou encore d'aménager les horaires à l'occasion du mois de jeûne. Mais, si cette circulaire constituait une avancée certaine pour les conditions d'exercice du culte musulman, celle-ci souffrait d'une application très inégale au sein des différentes enceintes militaires. Elle fut trop souvent tributaire des possibilités logistiques et matérielles ou parfois du bon vouloir des chefs de corps concernés.

La recherche relative aux militaires issus de l'im- 
migration commanditée par le ministère de la Défense en 2005, soit plus d'une décennie après la mise en place de la circulaire, dressait un constat quasi similaire. La facilitation de la pratique religieuse était très variable d'un régiment à l'autre et, de manière globale, peu d'évolutions avaient été constatées depuis la période de la conscription ${ }^{10}$. Les déficits relevés par le rapport Biville perduraient encore en de nombreux endroits et de nombreux régiments fonctionnaient selon une logique de " bricolages » mobilisant dans certains cas l'intervention des aumôniers catholiques et surtout israélites en raison de certaines proximités alimentaires (casher/halal).

En résumé, les rares expertises consacrées aux militaires issus de l'immigration ont mis en lumière l'existence de tensions directement liées à l'insuffisante prise en compte des besoins de militaires français de confession musulmane. Celles-ci étaient d'autant plus relevées par ces derniers que l'institution affiche une relative tolérance à l'expression de la foi et de la tradition culturelle catholique. Le déficit de prise en compte des demandes des musulmans générait un phénomène de repli identitaire potentiellement néfaste pour la cohésion et le moral des troupes. Par ailleurs, pour le haut-commandement, ce repli constituait également une source de questionnements sécuritaires dans un contexte sociétal marqué par une plus grande méfiance à l'égard de la religion islamique. Avec la fin des années 1990, on observe une certaine évolution de la "sociologie des actes terroristes ». À l'opposé des attentats des années 1980, essentiellement réalisés par des groupuscules étrangers, les actes terroristes des années 1990 et 2000 ont été commis par des individus largement socialisés dans des pays occidentaux. Les auteurs des attaques du 11 septembre 2001 avaient effectué des cursus universitaires dans des établissements occidentaux et certains des protagonistes de l'attentat de Londres étaient des citoyens britan- niques scolarisés en Grande-Bretagne, pleinement insérés dans le marché de l'emploi local. Ces différents attentats perpétrés au cœur des démocraties occidentales ont contribué à développer une suspicion à l'égard des segments de population issus de l'immigration et à accréditer une grille d'interprétation fondée sur la figure de l'ennemi intérieur ${ }^{11}$.

\section{Ajustement et formatage de l'islam à travers l'aumônerie militaire}

Calquée sur le modèle des trois aumôneries militaires existantes (catholique, protestante, israélite), l'aumônerie militaire musulmane vient mettre fin à un traitement différencié qui s'apparentait à une forme de discrimination institutionnelle. Sa mise en place vient ainsi réparer la situation jusque-là anticonstitutionnelle de l'État. Sans disposer de statistiques officielles sur la présence numérique des musulmans au sein des armées, les autorités s'accordent à penser qu'ils représentent, à l'instar de la société civile, la deuxième religion au sein de l'institution. Cette assertion difficilement vérifiable s'appuie essentiellement sur l'expérience des aumôniers et des chefs de corps rencontrés au cours de nos enquêtes. Outre les données collectées à travers différentes études ${ }^{12}$, la concrétisation du projet d'aumônerie musulmane s'est également appuyée sur le rapport du lieutenant-colonel Miloud Ait Hocine, en retraite et rappelé pour être nommé chargé de mission par le chef d'état-major des armées (Cema). À travers son étude réalisée en 2005, celui-ci a recueilli les demandes exprimées par les militaires musulmans sur le terrain. Conformément aux textes en vigueur, c'est le ministre de la Défense qui a nommé, en juin 2006, l'aumônier en chef Abdelkader Arbi sur proposition des autorités religieuses (Conseil français du culte musulman, CFCM). Car, si les organisations religieuses 
n'ont pas le pouvoir de nomination des aumôniers en chef, les textes prévoient néanmoins que le ministre en charge des armées doit sélectionner un candidat parmi ceux qu'elles proposent. Les autres aumôniers sont nommés par le ministre de la Défense sur proposition de l'aumônier militaire en chef de leur culte ${ }^{13}$. Recrutés avec un niveau minimal baccalauréat, les aumôniers doivent détenir la nationalité française et avoir un casier judiciaire vierge. Leurs compétences doivent s'inscrire à un double niveau, théologique et sociétal. Selon cette logique, ils doivent simultanément détenir de solides connaissances religieuses sur la religion musulmane mais également être en mesure d'analyser et de comprendre les grands enjeux qui traversent la société française. Ils exercent les mêmes missions que leurs collègues catholiques, protestants et israélites. L'article 1 du décret $n^{\circ} 247$ du 16 mars 2005 rappelle que les ministres des différents cultes "assurent le soutien religieux des personnels de la défense qui le souhaitent dans les lieux où les armées et formations rattachées exercent leurs missions ». Il précise également qu'ils "peuvent être consultés par le commandement dans leur domaine de compétences ${ }^{14}$ ». Leur positionnement intermédiaire entre fonction militaire et fonction religieuse sert également à apaiser les tensions susceptibles de se produire au sein des unités.

Faisant référence au conflit du Moyen-Orient, l'ex-chef d'état-major de l'armée de terre (Cemat) Bruno Cuche évoque explicitement ce rôle de modération œecuménique attribué aux différentes aumôneries : "Je crois également aux vertus modératrices des aumôneries pour guider les militaires croyants de toute confession à pratiquer leur foi dans le respect des principes du statut général et dans le sens de l'intérêt militaire. Car les armées, émanation de la nation, reflètent plus que toute autre institution sa diversité sociologique. Elles sont donc potentiellement exposées à des tendances centrifuges que cristallise, plus particulièrement à notre époque, la situation de guerre au Moyen-Orient, berceau des trois grandes religions autour desquelles se dessinent les principales lignes de fracture politique ${ }^{15}$. " Par leur posture, les aumôniers participent de la cohésion des troupes, en apportant un soutien moral et psychologique aux soldats, notamment durant les opérations extérieures, périodes cristallisant le plus de doutes et de questionnements. L'une des originalités de leur statut vient également du fait qu'ils n'ont « ni rang ni grade ». Ils sont assimilés à des officiers, ce qui leur permet de s'adresser à tout le monde et d'avoir ainsi une grande liberté de circulation au sein de l'institution. Mais, comme le souligne Xavier Boniface, cette absence de grade traduit peut-être également le souci de ne pas vouloir leur donner trop d'influence ${ }^{16}$. Le contrôle des armées sur le fonctionnement de l'aumônerie s'effectue également à travers la sélection des différents candidats. Chaque personne recrutée fait l'objet d'une enquête interne de la Direction de la protection et de la sécurité de la défense (DPSD) qui évalue la qualité et le profil de tous les candidats. Il s'agit de prévenir l'éventuelle intégration de profils jugés « radicaux » ou dont les idées seraient trop éloignées des valeurs républicaines françaises. Au-delà de ces règles de fonctionnement, il est intéressant de constater que les activités de l'aumônerie se calquent progressivement sur des pratiques existantes. Ainsi, conformément à l'aumônerie catholique ou protestante qui organise depuis très longtemps des sorties religieuses, l'aumônerie musulmane organise désormais un pèlerinage annuel à La Mecque pour ses militaires ainsi que les membres de leurs familles. Par ailleurs, il conviendra d'observer comment l'aumônerie musulmane, dont l'institutionnalisation est toute récente, relèvera le défi de puiser dans le corpus de la jurisprudence islamique les préceptes qui viendront, une fois affichés, renforcer les valeurs universelles de l'intérêt général prônées par l'armée. Il s'agira pour elle de se conformer aux valeurs d'inspiration judéo-chrétiennes qui imprègnent la culture militaire des armées françaises. Les exi- 
gences déontologiques du soldat, l'esprit de sacrifice ou encore le respect du droit international fondé sur la dignité humaine et la paix entre les peuples puisent en partie leurs racines dans l'héritage culturel chrétien. Des penseurs tels que saint Thomas d'Aquin ou saint Augustin constituent des sources d'inspiration et des références importantes dans le droit international de la guerre, notamment pour ce qui concerne, par exemple, la limitation des souffrances causées par les conflits (jus ad bellum/jus in bello). Ce legs culturel est assumé et même revendiqué par les acteurs militaires. Le chef d'état-major souligne dans ce sens : "Notre armée ne réfute pas la longue tradition qui l'a unie à l'ordre religieux, bien plus elle l'assume et l'intègre dans tout ce qui la structure. L'ordre surnaturel (la Transcendance) a été converti en un ordre supérieur naturel et républicain, qu'exaltent nos valeurs, nos traditions, notre cérémonial ${ }^{17}$. " À cet égard, il sera intéressant d'analyser le mode de sélection des sources scripturaires musulmanes qui sera opéré par les acteurs de l'aumônerie pour démontrer que les valeurs généralistes d’inspiration judéo-chrétienne ont leur équivalent au sein de l'islam.

\section{Militaires français et musulmans: quelles pratiques?}

L'analyse formelle du " croire musulman » du groupe de militaires de notre enquête ${ }^{18}$ dessine un visage original faisant ressortir a minima quatre caractéristiques. D'une part, les récits relatifs aux modalités de leur croyance traduisent un fort processus d'individualisation. Celui-ci a été précisément décrit par Danièle Hervieu-Léger dans ses analyses de la modernité religieuse. Celles-ci mettent en exergue l'idée que les acteurs s'inscrivent de moins en moins dans des systèmes contraignants et constitués en dehors d'eux-mêmes. La tendance est désormais à "l'éclatement des systèmes de croyance et la dissémination des petits récits croyants que les individus produisent eux-mêmes à partir de leurs aspirations, de leurs intérêts, de leurs dispositions, de leurs expériences ${ }^{19}$ ". Pour l'auteure, cette évolution se traduit par la multiplication de syncrétismes et de "bricolages individuels » qui continuent de se réaliser en référence aux grands récits issus des traditions religieuses, à l'intérieur desquels les individus puisent comme dans une bibliothèque de symboles et de formes religieuses. Ils deviennent acteurs en choisissant et en s'appropriant un certain type de croyance qu'ils créent et modulent en fonction de ce qui fait «sens » pour eux.

Pour paraphraser le titre d'un ouvrage ${ }^{20}$, on pourrait dire qu'au moment de la réalisation de notre enquête, "Monsieur Islam n'existe pas » au sein de l'institution militaire. Nos constats corroborent de manière significative les notions de "pratiques religieuses bricolées » relevées par Catherine de Wenden et Christophe Bertossi. Ces derniers soulignent toute l'hétérogénéité des pratiques qui s'articulent autour de plusieurs pôles: " Être musulman dans l'armée englobe un large spectre de pratiques, allant de ceux qui se considèrent comme musulmans non pratiquants, voire simplement "d'origine musulmane", à ceux qui pratiquent leur religion à différents niveaux de rigueur, du respect des restrictions alimentaires au ramadan, jusquà la prière. Ainsi, au niveau des pratiques religieuses, un véritable bricolage s'observe, fait de beaucoup d'assemblages possibles et qui s'organise autour de trois pôles : les restrictions alimentaires et l'alcool, le ramadan et les prières ${ }^{21}$. » Comme le relèvent les auteurs, ces trois pôles fonctionnent selon une logique de hiérarchisation. La réalisation des prières quotidiennes traduit une implication plus intense dans la religion que l'observation du mois de jeûne du ramadan. De même, le respect des interdits alimentaires 
(porc et alcool) apparait comme la pratique la plus basique des prescriptions religieuses et se justifie parfois comme la perpétuation d'une simple habitude culturelle. Concrètement, ces trois pôles de pratique s'articulent selon des combinaisons multiples, car ils entrent en interaction avec une série de contraintes objectives qui vont orienter les possibilités d'application. Celles-ci restent fortement tributaires de la latitude qu'autorisent l'environnement matériel, la spécialisation professionnelle ou le contexte opérationnel dans lequel les militaires se situent.

La deuxième caractéristique que nous avons pu observer a trait à la définition et à l'interprétation donnée par les musulmans de leur pratique religieuse. Les entretiens font ressortir une présentation et une définition de leur religion davantage orientée sur la dimension éthique et morale de la foi que sur son versant théologique ou politico-juridique. Plus que sur les aspects proprement religieux ou dogmatiques, l'islamité est avant tout présentée comme un code comportemental entrant en forte congruence avec les valeurs militaires. C'est ce que rapporte Messaoud dans ses propos : «Être musulman je dirais que c'est d'abord être sérieux et rigoureux dans sa vie de tous les jours. C'est les mêmes valeurs que l'armée. » (Messaoud, sous-officier, 25 ans). Pour d'autres, l'islam est une source de plusvalue pour l'occupation du métier de militaire. Ainsi, selon Tarek, le respect des prescriptions religieuses islamiques constituerait une ressource supplémentaire dans l'accomplissement de son métier : «Pour moi, être musulman, c'est même un plus pour quelqu'un qui veut être militaire. Tu ne bois pas, et tu ne fumes pas donc tu maintiens ton corps en forme, c'est une bonne hygiène. Tu apprends la solidarité avec ton groupe. Tout ça, c'est des valeurs de l'islam et je pense ça ne peut être qu’une force dans les métiers de l'armée. »

Le troisième constat s'inscrit dans une logique de continuité avec la deuxième caractéristique, et concerne la déconnexion de nos militaires d'avec les différentes instances de représentation du culte musulman français. À l'exception d'un militaire aumônier que nous avons interviewé, la plupart des militaires de culture musulmane méconnaissaient l'existence des différentes structures de représentation cultuelle. Une très petite minorité connaissait l'existence du CFCM, tout en se positionnant en décalage avec ses représentants, jugés illégitimes et parfois péjorativement qualifiés de "blédards ${ }^{22}$ ". À cette faible identification aux instances censées les représenter, s'ajoute enfin une forte connotation affective associée au « croire musulman ». Être musulman, c'est dans le même temps maintenir une tradition et une lignée familiale, mais cela renvoie également à une palette d'expériences dotées d'une forte charge affective et sentimentale. Parmi elles, figurent aussi bien les festivités religieuses que les parfums du pays d'origine et les vacances estivales annuelles, qui forment autant d'occasions de raviver cet héritage culturel en compagnie de la famille élargie. Cette dimension culturelle et festive s'observe notamment durant le mois de ramadan, où le sens ascétique originel de la pratique se dévoie au profit d'une approche orientée autour de la convivialité et du plaisir partagé : "Le ramadan, pour moi c'est surtout le plaisir dêtre en famille le soir et partager un bon moment autour d'un bon repas. " (Noor, sous-officier, 27 ans). On retrouve cette volonté de recréer une ambiance collective au sein même des bases militaires : "On recrée un peu l'ambiance de l'if$\operatorname{tar}$ (rupture du jeûne) dans notre base militaire. On se retrouve tous dans une salle et on passe un bon moment ensemble. Je l'ai déjà fait toute seule, mais ça n'a pas de sens. Même le soir quand tu manges de façon solitaire, tu sens qu'il manque quelque chose. » (Karima, sousofficier, 30 ans).

Il faut également noter que la diversité des modes de pratiques musulmanes se conjugue à la pluralité des conceptions des fonctions de l'institution militaire. En effet, les armées sont loin de représenter un bloc monolithique en termes d'auto-définition de leur rôle au sein de l'espace social, surtout depuis le passage à la professionnalisation intégrale. Ainsi, certains cadres militaires demeurent attachés dans leurs représentations à une armée intégratrice des- 
tinée à transformer l'individu en citoyen et qui fait donc largement écho à sa mission historique depuis la Troisième République. D'autres se dissocient de cette philosophie originelle d'intégration fondée sur le " colour blindness", en mettant l'accent sur les profondes transformations organisationnelles et la nécessité d'être avant tout efficace d'un point de vue organisationnel. Ces disparités tendent naturellement à influer sur les perceptions de l'altérité à l'intérieur de la sphère militaire ${ }^{23}$.

\section{Conclusion}

L'instauration en 2006 de l'aumônerie musulmane au sein des armées françaises constitue une initiative historique qui, de fait, met fin à une situation d'iniquité à l'égard des militaires partageant cette confession. L'analyse des facteurs qui ont favorisé son émergence montre que ceux-ci sont protéiformes. Ils sont à un premier niveau d'ordre fonctionnel. Les nombreux déficits identifiés depuis les années 1990 par les rapports internes des armées ont permis de mettre en lumière les incidences négatives qu'ils avaient en termes de cohésion interne des unités. Ce constat est devenu encore plus prégnant lorsque l'institution s'est totalement professionnalisée en 1996. Confrontée à des impératifs de recrutement massif, elle a dû développer et approfondir sa réflexion sur l'accueil et la gestion de ces soldats désormais volontaires. L'émergence de l'aumônerie est également à mettre en parallèle avec les tendances sociétales des années 2000 et le développement en France des enjeux liés aux questions d'égalité des chances et de diversité. La création en mai 2003 du Conseil français du culte musulman (CFCM) a également contribué à activer la mise en place de cette instance. Comme on a pu le constater au fil de l'analyse, le statut des aumôniers militaires dans le contexte français demeure très particulier eu égard au régime de séparation des Églises et de l'État défendu par la loi de 1905 Bénéficiant du statut de militaires, ils sont de fait rémunérés par l'État pour assurer une assistance religieuse auprès des unités au sein desquelles ils sont affectés, cet aspect dérogatoire étant spécifié dans l'article 2 de ladite loi afin de permettre aux citoyens évoluant dans des structures fermées d'accomplir leurs obligations religieuses. Simultanément fidèles à leur patrie et à leur religion, les aumôniers occupent une position originale au croisement de la sphère religieuse et de la sphère militaire. Assurant le soutien moral et religieux des soldats, ils représentent aussi une précieuse source d'information pour le commandement. Bien que récente, il est possible d'identifier les modalités par lesquelles cette nouvelle aumônerie ajuste progressivement son mode de fonctionnement sur les pratiques de celles déjà existantes. Encadré par le ministère de la Défense, ce processus de formatage est particulièrement efficace.

Enfin, on peut s'interroger sur la symbolique et les incidences sociétales de l'émergence de cette aumônerie. De même que les aumôniers militaires catholiques ont, tout au long du XXe siècle, contribué à apaiser les relations très conflictuelles entre l'Église et l'État français ${ }^{24}$, on peut se demander si aujourd'hui l'aumônerie militaire musulmane ne joue pas ce même rôle symbolique au sein de la société française. Dans un contexte de méfiance voire de suspicion à l'égard des citoyens de confession musulmane et le développement d'une grille de lecture essentialiste sur l'islam25, ce processus d'institutionnalisation vient aussi rappeler à la majorité des citoyens l'engagement bien réel de ces Français au service de la République. Même si la légitimité des interventions militaires menées par les armées françaises au cours de cette dernière décennie (Afghanistan, Mali, Libye, etc.) cristallise de profonds questionnements et clivages tant du point de vue des communautés musulmanes que de l'opinion publique française dans sa globalité. 